\section{Assessing prospective memory beyond experimental tasks}

\author{
Mathieu Hainselin (1D, Yannick Gounden (1) and Geoffrey Blondelle (1)
}

We read with great interest the Review by Henry on prospective memory (PM) impairment in neurological disorders (Henry, J. D. Prospective memory impairment in neurological disorders: implications and management. Nat. Rev. Neurol. https://doi.org/ 10.1038/s41582-021-00472-1 (2021)) $)^{1}$. The author outlines the frequent occurrence of PM impairments in various neurological disorders, with a focus on PM assessment. Specifically, the author proposes that "self-report measures often correlate weakly with objective assessments" and "single-item PM tests have lower reliability and sensitivity than clinical batteries."

Recent literature reviews have highlighted the flaws of clinical batteries and emphasized the relevance of questionnaires and single-item PM tests. In a systematic and meta-analytic review of PM assessment tools ${ }^{2}$, Blondelle and colleagues highlighted the lack of standardized neuropsychological evaluation in clinical practice. The authors showed that many PM tools lacked normative data, cut-off scores for diagnostic purposes, qualitative scoring, parallel versions and/or ecological validity. Although translated versions of some tools exist, there is a distinct lack of cultural adaptation in non-WEIRD (Western, educated, industrialized, rich and democratic) populations $^{3}$, and progress in this area will rely on following specific guidelines for test development ${ }^{4}$. Performance-based measures (so-called objective assessments) are valuable for clinical practice, but we should not forget the work needed to make these tools available and relevant to each patient.

With the current focus on performancebased measures, it would be regrettable if single-item PM tests were disregarded. Although we acknowledge that the Key Task is of limited clinical interest, the Envelope Task is interesting to spot patients with dementia and is sensitive enough to detect difficulties associated with amnestic mild cognitive impairment ${ }^{6}$. In these scenarios at least, continued incorporation of this measure into routine clinical practice could be warranted.

We agree with Henry's point of view regarding the weak correlation between self-report and performance-based measures, and that the former measures "should supplement rather than replace a formal behavioural assessment."
However, a recent scoping review ${ }^{7}$ argues that self-report and informant-report PM measures evaluate different constructs from those targeted by performance-based tests, and can also sometimes aid the distinction between individuals with and without PM impairment ${ }^{7}$. Sugden et al. also pointed out the importance of self-report measures to assess the impact of interventions. As such, self-report and informant-report measures seem to represent metacognitive measures of the concerns of individuals about their PM ability rather than measures of PM performance per se.

Finally, in addition to the neurological disorders discussed in the Henry Review ${ }^{1}$, researchers and clinicians should be aware that PM impairments are also found in patients with rare conditions such as spina bifida meningomyelocele ${ }^{8}$ and transient global amnesia?.

There is a reply to this letter by Henry, J. D. Reply to: Assessing prospective memory beyond experimental tasks. Nat. Rev. Neurol. https:// doi.org/10.1038/s41582-021-00501-z (2021).
Mathieu Hainselin (D) ${ }^{凶}$, Yannick Gounden (D and Geoffrey Blondelle iD CRP-CPO, UR UPJV 7273, Université de Picardie Jules Verne, Amiens, France.

凶e-mail: mathieu.hainselin@u-picardie.fr https://doi.org/10.1038/s41582-021-00499-4

1. Henry, J. D. Prospective memory impairment in neurological disorders: implications and management. Nat. Rev. Neurol. https://doi.org/10.1038/s41582021-00472-1 (2021).

2. Blondelle, G., Hainselin, M., Gounden, Y. \& Quaglino, V. Instruments measuring prospective memory: a systematic and meta-analytic review. Arch. Clin. Neuropsychol. 35, 576-596 (2020).

3. Henrich, J., Heine, S. J. \& Norenzayan, A. Most people are not WEIRD. Nature 466, 29 (2010).

4. American Educational Research Association, American Psychological Association \& National Council on Measurement in Education. Standards for Educational and Psychological Testing (American Educational Research Association, 2014)

5. Huppert, F. A., Johnson, T. \& Nickson, J. High prevalence of prospective memory impairment in the elderly and in early-stage dementia: findings from a population-based study. Appl. Cogn. Psychol. 14 S63-S81 (2000)

6. Lee, S. et al. The contribution of prospective memory performance to the neuropsychological assessment of mild cognitive impairment. Clin. Neuropsychol. 30 131-149 (2016).

7. Sugden, N., Thomas, M. \& Kiernan, M. A scoping review of the utility of self-report and informantreport prospective memory measures. Neuropsychol. Rehabil. https://doi.org/10.1080/09602011.2021. 1875851 (2021)

8. Dennis, M., Nelson, R., Jewell, D. \& Fletcher, J. M. Prospective memory in adults with spina bifida. Childs Nerv. Syst. 26, 1749-1755 (2010).

9. Hainselin, M. et al. Can we remember future actions yet forget the last two minutes? Study in transient global amnesia. J. Cogn. Neurosci. 23, 4138-4149 (2011).

Competing interests

The authors declare no competing interests.

\title{
Reply to: Assessing prospective memory beyond experimental tasks
}

\section{Julie D. Henry (D)}

Two statements from my recent Review on prospective memory (PM) impairment (Henry, J. D. Prospective memory impairment in neurological disorders: implications and management. Nat. Rev. Neurol. https://doi.org/ 10.1038/s41582-021-00472-1 (2021)) $)^{1}$ are highlighted as warranting discussion by Hainselin et al. (Hainselin, M., Gounden, Y. \& Blondelle, G. Assessing prospective memory beyond experimental tasks. Nat. Rev. Neurol. https://doi.org/10.1038/s41582021-00499-4 (2021)) $)^{2}$, namely, "self-report measures often correlate weakly with objective assessments" and "single-item PM tests have lower reliability and sensitivity than clinical batteries." Hainselin et al. argue that "recent literature reviews have highlighted the flaws of clinical batteries and emphasized the relevance of questionnaires and single-item PM tests."
As I discussed ${ }^{1}$, self-report scales provide valuable insights into a patient's own perspective, but, because they often correlate weakly with objective assessments, they should supplement rather than replace a formal behavioural assessment. This viewpoint aligns completely with the following conclusions from the review that Hainselin et al. ${ }^{2}$ themselves cite ${ }^{3}$ : "this review found that selfand informant-report measures have relatively weak relationships with performance-based measures of PM. Some limited evidence of self-report and informant-report measures being able to detect PM impairments and monitor intervention outcomes is reported. As such, these measures are most suitable for the measurement of individuals' concerns and beliefs about their PM ability and the impact of PM failures on their lives rather than measures of PM ability itself." 